\title{
Vibration Control of Systems in Presence of Hard Nonlinearities
}

\author{
Mario Terzo, ${ }^{1}$ Chia-Ming Chang, ${ }^{2}$ Zi-Qiang Lang, ${ }^{3}$ and Salvatore Strano ${ }^{1}$ \\ ${ }^{1}$ Department of Industrial Engineering, University of Naples Federico II, 80125 Naples, Italy \\ ${ }^{2}$ Department of Civil Engineering, National Taiwan University, Taipei 10617, Taiwan \\ ${ }^{3}$ Department of Automatic Control and Systems Engineering, University of Sheffield, Mappin Street, Sheffield S1 3JD, UK \\ Correspondence should be addressed to Mario Terzo; m.terzo@unina.it
}

Received 4 November 2015; Accepted 5 November 2015

Copyright (C) 2016 Mario Terzo et al. This is an open access article distributed under the Creative Commons Attribution License, which permits unrestricted use, distribution, and reproduction in any medium, provided the original work is properly cited.

Vibration phenomena involve a wide range of engineering systems and structures such as buildings, vehicles, aircrafts, bridges, and electronic components. Due to the undesired effects of the vibration induced motions, the vibration control is a theme on which many researchers are focusing their attention in the recent years. The main scope of the vibration control is the suppression or, at least, the attenuation of the undesirable vibrations by means of passive, semiactive, or active devices. Hard nonlinearities can be found in vibrating systems because of inherent system nonlinear properties and/or the presence of dampers, stiffness elements, and actuators which are often characterized by hysteresis, dead zone, and so forth. This special issue intends to provide an upto-date overview of recent advances in the vibration control of systems characterized by hard nonlinearities.

In the paper "Modelling of Hysteresis in Vibration Control Systems by means of the Bouc-Wen Model" by C.-M. Chang et al., applications of the Bouc-Wen model in vibration control devices are reviewed. The paper "Novel Distributed PZT Active Vibration Control Based on Characteristic Model for the Space Frame Structure" by H. Zhong et al. presents a novel distributed PZT control strategy based on characteristic model for space frame structure. The paper "Recent Advances in Bidirectional Modeling and Structural Control" by S. Paul et al. gives an overview of building structure modeling and control under bidirectional seismic waves. In the paper "Optimal Trajectory Planning and Linear Velocity Feedback Control of a Flexible Piezoelectric Manipulator for Vibration Suppression" by J. Lou et al., an optimal trajectory planning approach is proposed and applied to a flexible piezoelectric manipulator system. The paper "Nonlinear Seismic Behavior of Different Boundary Conditions of Transmission Line Systems under Earthquake Loading" by L. Tian and X. Gai investigates nonlinear seismic behaviors of different boundary conditions of transmission line system under earthquake loading. The paper "A Water Hammer Protection Method for Mine Drainage System Based on Velocity Adjustment of Hydraulic Control Valve" by Y. Kou et al. proposes a water hammer protection method based on velocity adjustment of hydraulic control valve to deal with the problem of valveclosing water hammer in mine drainage system. In the paper "Novel Hyperbolic Homoclinic Solutions of the HelmholtzDuffing Oscillators" by Y.-Y. Chen, a new homoclinic solution of the Helmholtz-Duffing oscillator is presented. The paper "Modeling and Simulation of Flexible Transmission Mechanism with Multiclearance Joints for Ultrahigh Voltage Circuit Breakers" by F. Meng et al. proposes a planar rigid-flexible coupling dynamic model of high-speed multilink transmission mechanisms with clearance for ultrahigh voltage circuit breaker. In the paper "Experimental and Theoretical Study on Influence of Different Charging Structures on Blasting Vibration Energy" by W. Gu et al., a study is designed to deduce the law of different charging structures' influence on blasting vibration energy of far region of blasting and provide reference for controlling side effects of blasting vibration and designing parameters of charging structure. The paper "Nonlinear Dynamics Analysis of the Semiactive Suspension System with Magneto-Rheological Damper" by H. Zhang et al. examines dynamical behavior of a nonlinear oscillator which models a quarter car forced by the road profile. The magneto-rheological (MR) suspension system has been 
established, by employing the modified Bouc-Wen forcevelocity model of magneto-rheological damper.

Mario Terzo Chia-Ming Chang Zi-Qiang Lang Salvatore Strano 


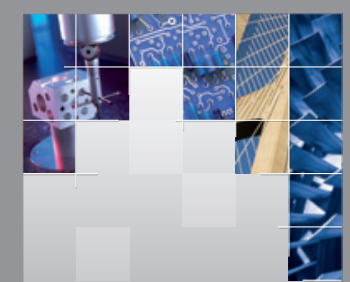

\section{Enfincering}
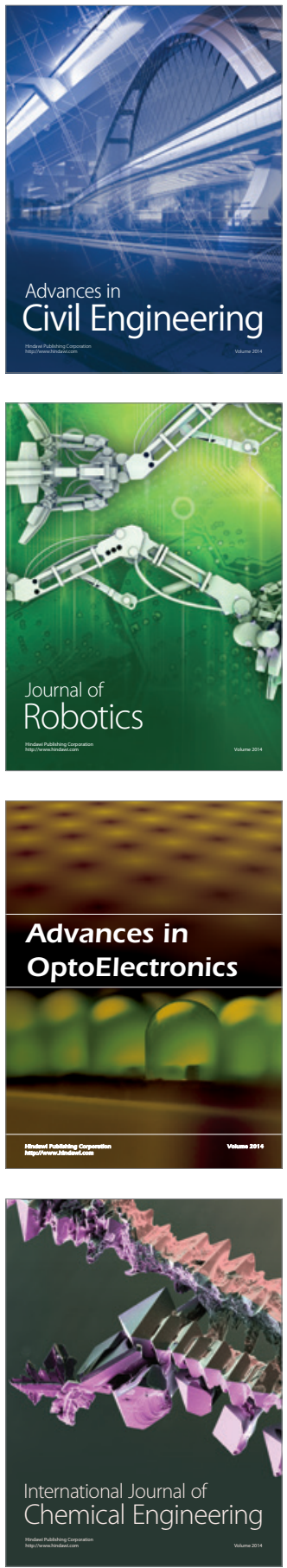

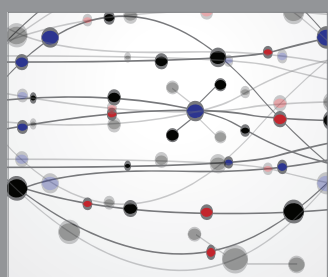

The Scientific World Journal

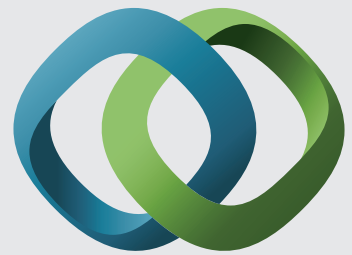

\section{Hindawi}

Submit your manuscripts at

http://www.hindawi.com
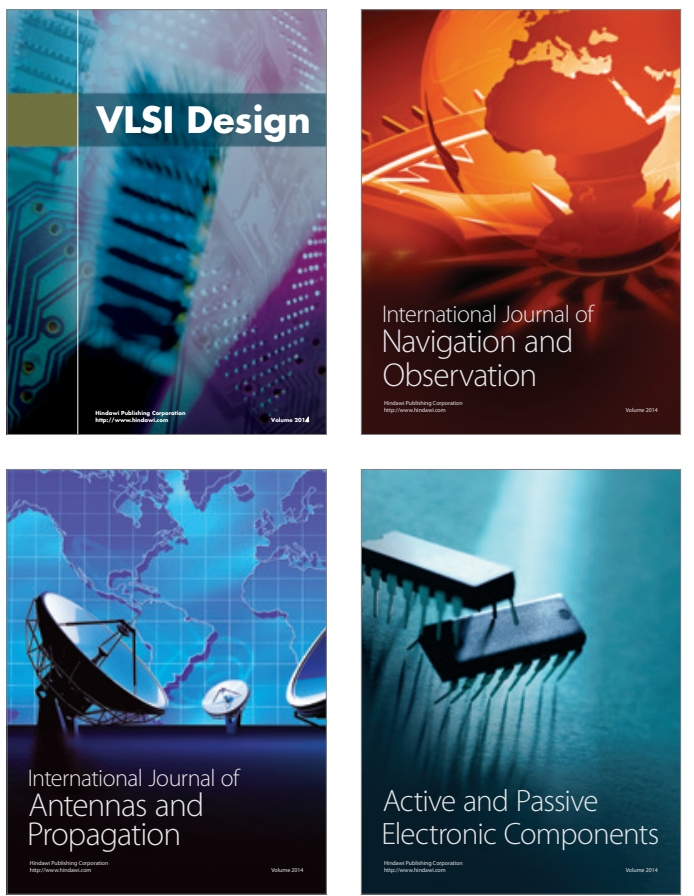
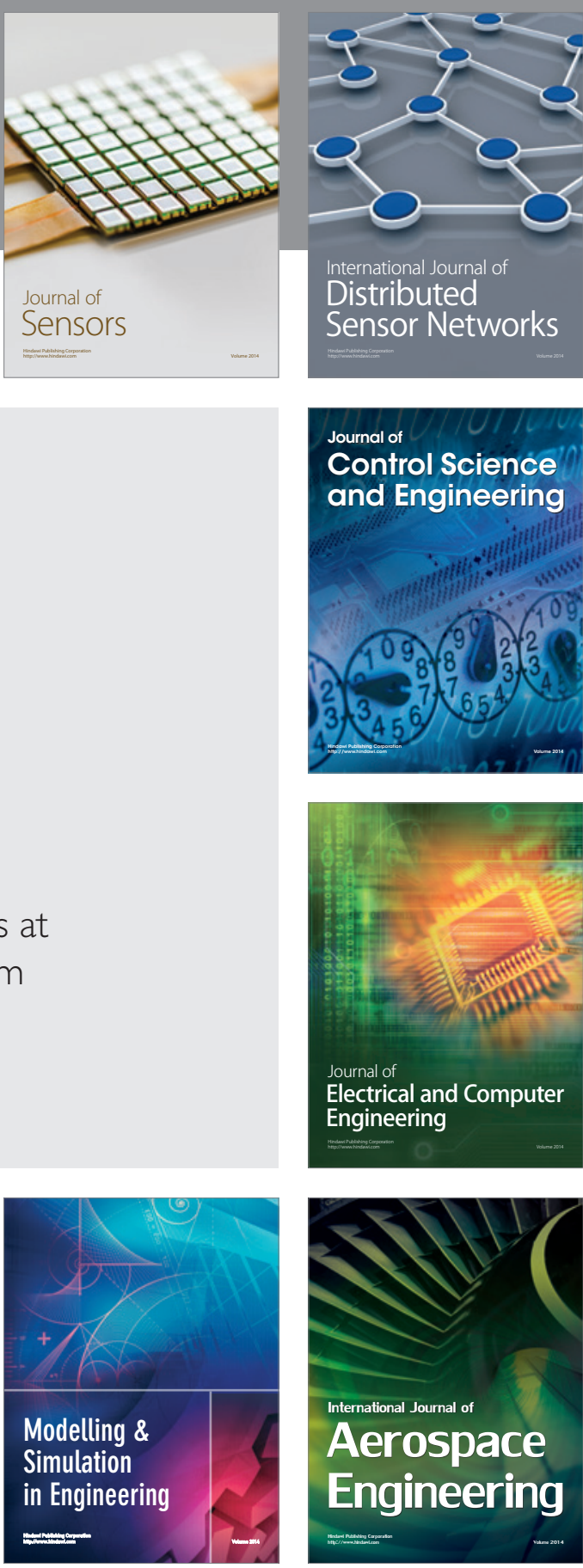

International Journal of

Distributed

Sensor Networks

Journal of

Control Science

and Engineering
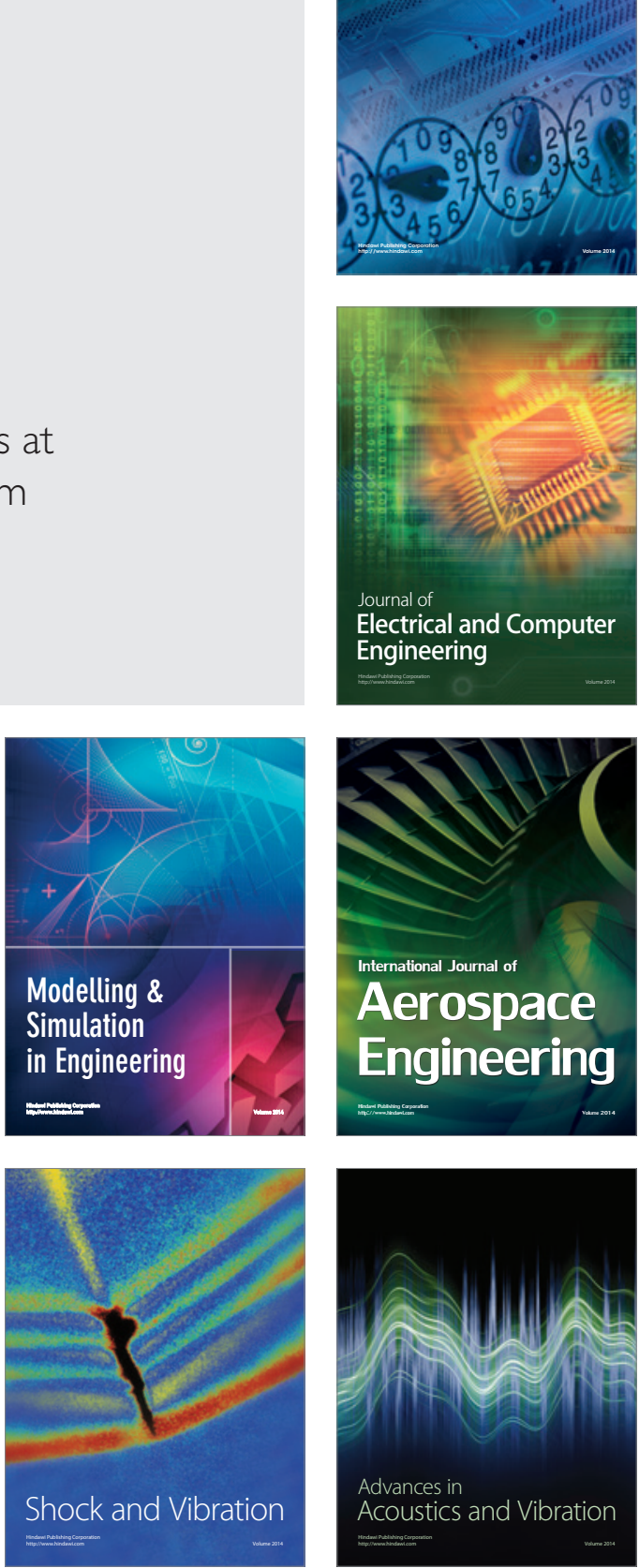